\title{
Effectiveness of different elicitors in inducing resistance in chilli (Capsicum annuum L.) against pathogen infection.
}

\begin{abstract}
The excessive application of pesticides for agricultural production in Malaysia has raised quite some concern about environmental safety and sustainability. To reduce environmental impact of pesticide overuse, there is an increasing interest in using different elicitors to induce disease resistance in crop. Chilli (Capsicum annuum L.), which is an important vegetable cum spice crop in Malaysia, was used to compare the effectiveness of two natural elicitors (jasmonic acid and salicylic acid) with conventional pesticide application as control. The experimental results indicated that pesticide-treated plants showed rapid reduction in disease severity after application while elicitors perform slowly and its effectiveness increase gradually over time. Among the tested elicitors, jasmonic acid was found most effective regarding disease severity and yield of chilli compared with salicylic acid. Although used elicitors was not best performing treatment compared with conventional pesticide, some physiological parameters (relative chlorophyll content, chlorophyll fluorescence and photosynthesis rate) and disease severity in chilli plants treated with jasmonic acid was very close to conventional pesticide. Therefore, jasmonic acid could be a potential elicitor for inducing disease resistance in chilli and salicylic acid may not an appropriate elicitor for chilli.
\end{abstract}

Keyword: Chilli; Disease resistance; Jasmonic acid; Salicylic acid. 